\title{
Quantum Transport in Two-Channel Fractional Quantum Hall Edges
}

\author{
K. Imura and N. Nagaosa \\ Department of Applied Physics, University of Tokyo, Bunkyo-ku, Tokyo 113, Japan
}

(March 24, 2018)

\begin{abstract}
We study the effect of backward scatterings in the tunneling at a point contact between the edges of a second level hierarchical fractional quantum Hall states. A universal scaling dimension of the tunneling conductance is obtained only when both of the edge channels propagate in the same direction. It is shown that the quasiparticle tunneling picture and the electron tunneling picture give different scaling behaviors of the conductances, which indicates the existence of a crossover between the two pictures. When the direction of two edge-channels are opposite, e.g. in the case of MacDonald's edge construction for the $\nu=2 / 3$ state, the phase diagram is divided into two domains giving different temperature dependence of the conductance.
\end{abstract}

72.10.-d, 73.20.Dx, 73.40.Hm 


\section{INTRODUCTION}

It has been discovered that the edge mode of a fractional quantum Hall (FQH) system is described as a chiral Tomonaga-Luttinger (TL) liquid [1]. When one makes a point contact between the edges, backward scatterings become possible only near that point contact. Such a system is expected to be described as a TL model with a scattering potential at $x=0$ [2].3]. For a $\nu=1 / 3$ state, the model predicts a $T^{4}$ dependence of the tunneling conductance near the zero temperature [4 6] which is consistent with the recent experiment [7]. The result does not rely upon whether we start the renormalization group $(R G)$ analysis with the picture where quasiparticles tunnel between the edges through the bulk FQH liquid (quasiparticle tunneling picture) or the picture where electrons tunnel through the vacuum between the edges of two condensates separated by the tunnel effect (electron tunneling picture) [8].

To describe the bulk FQH liquids with a filling factor $\nu \neq \frac{1}{2 k+1}$ hierarchy constructions are introduced 9 14. At a given filling one can easily propose many hierarchical constructions. When we apply the above discussion on the edge mode to the hierarchy states, we encounter a completely novel situation. Let us consider a two-terminal geometry where the bulk FQH liquids between the left and right terminals have upper and lower edges. Each of the edge branches consists of more than two channels. In this case the tunneling at apoint contact as well as the interaction between the channels become important for the quantum transport at the edges. For $\nu=2 / 5$ and $2 / 3$ there are two channels of the edge modes [5] which are specified by a set of filling factors $\left(\nu_{1}, \nu_{2}\right)$. However the possibility that there are more edge channels cannot be excluded [15, 16].

For $\nu=2 / 3$ two constructions with $\left(\nu_{1}, \nu_{2}\right)=(1,-1 / 3)$ and $(1 / 3,1 / 3)$ have been proposed, which correspond respectively to sharp [17] and smooth confinement [16]. In the former (latter) construction two edge modes in a same branch propagate in the opposite (same) direction. Recent numerical calculations based on the composite Fermion approach in the Hartree approximation confirmed that there is a transition from the MacDonald's (former) picture to the Beenakker's (latter) one as the confinement is relaxed 15,18.

So far we have not taken into account the spin degrees of freedom since we have assumed that all the spins are polarized. However finite-size calculations of the Coulomb energy show that the unpolarized spin configuration is preferred when $\nu=2 / 5$ or $2 / 3$ [19]. The result is consistent with the experiment in tilted magnetic fields [20. Nevertheless we can realize the spin-polarized FQH states at arbitrary filling by applying the strong parallel magnetic field.

Wen has shown that the FQH state has gapless boundary excitations using the gauge invariance of the bulk effective theory [5]. In his derivation, low-energy effective action for the edge mode is determined only from the gauge invariance. In the appendix we give an alternative derivation. We describe the edge mode as a self-induced eigenmode of the bulk FQH state [21]. It turns out that the existence of Maxwell terms in the dual action of the bulk effective theory is essential for our derivation.

We study the effects of backward scatterings at a point contact using the effective action generalized for the two-channel edge modes. We concentrate on the analysis of $\nu=2 / 5$ and 2/3 FQH edges but our formulation can be applied to any two-channel FQH edges. We assume that the interaction between the electrons is short-ranged. The interaction between the upper and lower branches are neglected.

We consider both of the limits where the scattering potential is very weak and very strong. In the weak potential limit we start the RG analysis from the quasiparticle tunneling picture and study the scaling behavior of the deviation of the conductance from the quantized value. The situation corresponds to a "high" temperature. As the potential becomes strong, the 
tunneling through the potential barrier becomes rare and we can employ the dilute instanton gas approximation (DIGA). As the potential further increases, it is better to start with the electron tunneling picture. The question is whether or not the scaling behaviors of the tunneling conductance are smoothly connected from one picture to the other.

The plan of the paper is the followings. In Sec. II the model for the edge mode is introduced. In Sec. III the quasiparticle tunneling picture is explained, and the RG analysis in the weak and strong potential limit is given. Electron tunneling picture is studied in Sec. IV. Sec. V is devoted to discussion and conclusions.

\section{MODEL}

Our model describes a two-terminal Hall bar geometry where a two-dimensional electron system between the left and right terminals has upper and lower edges. Low-energy excitations of the system are the modes which are localized near the edges since bulk FQH state has an energy gap. In our model, edge modes of both upper and lower branches consist of two channels (1-channel and 2-channel). In the following,

(a) We assume that long-range Coulomb interactions are screened away and electrons in the FQH edges have only intra-branch short-range interactions.

(b) We consider only the backward scatterings at a point contact, since forward scatterings are relevant but do not open an energy gap [22], and since backward scatterings except at a point contact are forbidden as a result of momentum conservation.

(c) We also assume that the spins are fully polarized. This may not be true for $\nu=2 / 5$ and $2 / 3$. This issue will be discussed in future publications.

\section{A. Effective action for the tunneling}

The Lagrangian density (A7) in the appendix is the starting point of our following analysis. We generalize it to the two-channel case as

$$
\mathcal{L}_{I}=\sum_{I=1,2}\left[\frac{c_{I}}{8 \pi \nu_{I}}\left\{\left(\frac{\partial \phi_{I}^{(+)}}{\partial x}\right)^{2}+\left(\frac{\partial \phi_{I}^{(-)}}{\partial x}\right)^{2}\right\}+\frac{i}{4 \pi \nu_{I}} \frac{\partial \phi_{I}^{(+)}}{\partial \tau} \frac{\partial \phi_{I}^{(-)}}{\partial x}\right]
$$

Short-range electron-electron interactions have the form $V=V_{11}+V_{22}+2 V_{12}$ where $V_{I I}$ 's are intra-channel interactions: $V_{I I}=\pi g_{I I} \int d x\left\{\left(\rho_{I}^{(u)}(x)\right)^{2}+\left(\rho_{I}^{(l)}(x)\right)^{2}\right\}$ for $I=1,2$ and $V_{12}$ is the

inter-channel interaction: $V_{12}=\pi g_{12} \int d x\left\{\rho_{1}^{(u)}(x) \rho_{2}^{(u)}(x)+\rho_{1}^{(l)}(x) \rho_{2}^{(l)}(x)\right\}$. All $g_{I J}$ 's are positive since we consider the repulsive Coulomb interaction. The most general form of the edge action for the multi-channel FQH states has the form

$$
S_{0}=\sum_{I, J} \int d \tau d x\left\{\frac{1}{8 \pi} v_{I J}\left(\frac{\partial \phi_{I}^{(+)}}{\partial x} \frac{\partial \phi_{J}^{(+)}}{\partial x}+\frac{\partial \phi_{I}^{(-)}}{\partial x} \frac{\partial \phi_{J}^{(-)}}{\partial x}\right)+\frac{i}{4 \pi} K_{I J} \frac{\partial \phi_{I}^{(+)}}{\partial \tau} \frac{\partial \phi_{J}^{(-)}}{\partial x}\right\}
$$

In our two-channel model, since two matrices $K$ and $v$ are given by

$$
K=\left[\begin{array}{cc}
1 / \nu_{1} & 0 \\
0 & 1 / \nu_{2}
\end{array}\right], \quad v=\left[\begin{array}{cc}
v_{1} / \nu_{1} & g_{12} \\
g_{12} & v_{2} / \nu_{2}
\end{array}\right]
$$

where $v_{I}=c_{I}+\nu_{I} g_{I I}$, the action (2) reduces to the following special form 


$$
S_{0}=\frac{1}{\beta L} \sum_{\omega, k} \frac{k^{2}}{8 \pi} \phi^{T}(-\omega,-k) A(\omega, k) \phi(\omega, k)
$$

where $\phi^{T}=\left(\phi_{1}^{(+)}, \phi_{1}^{(-)}, \phi_{2}^{(+)}, \phi_{2}^{(-)}\right) . A(\omega, k)$ is a 4 by 4 matrix which has the form

$$
A=\left[\begin{array}{rr}
A_{1} & g_{12} I \\
g_{12} I & A_{2}
\end{array}\right]
$$

where $I$ is the $2 \times 2$ identity matrix, and

$$
A_{J}=\left[\begin{array}{rr}
a_{J} & -i b_{J} \\
-i b_{J} & a_{J}
\end{array}\right]
$$

with $a_{J}=v_{J} / \nu_{J}, b_{J}=\omega / \nu_{J} k$ for $J=1,2$. When we introduce backward scatterings between the edges by making a point contact, the system is described as the TL model with a scattering potential at $x=0$. Since here we do not need the detailed structure of the scattering potential $U[\phi(\tau, x=0)]$, we leave the analysis of it to the next section. All we need here is that the total action has the form $S=S_{0}+\int d \tau U[\phi(\tau, x=0)]$. The partition function $Z=\int \mathcal{D} \phi e^{-S}$ of the system can be written in terms of the field $\theta(\tau)=\phi(\tau, x=0)$ as

$$
Z=\int \mathcal{D} \theta \int \mathcal{D} \lambda \int \mathcal{D} \phi e^{-S_{0}[\theta, \lambda, \phi]-\int d \tau U[\theta(\tau)]}
$$

where

$$
S_{0}[\theta, \lambda, \phi]=S_{0}+i \int d \tau \lambda^{T}(\tau)(\theta(\tau)-\phi(\tau, x=0))
$$

with $\lambda^{T}=\left(\lambda_{1}^{(+)}, \lambda_{1}^{(-)}, \lambda_{2}^{(+)}, \lambda_{2}^{(-)}\right), \theta^{T}=\left(\theta_{1}^{(+)}, \theta_{1}^{(-)}, \theta_{2}^{(+)}, \theta_{2}^{(-)}\right)$by introducing the auxiliary field $\lambda(\tau)$ so that we may obtain $\theta(\tau)=\phi(\tau, x=0)$ after integrating out the $\lambda(\tau)$ 's. We perform successive integrations as $\int \mathcal{D} \lambda \int \mathcal{D} \phi e^{-S_{0}[\theta, \lambda, \phi]}=\int \mathcal{D} \lambda e^{-S_{0}[\theta, \lambda]}=e^{-S_{0}[\theta]}$ where $S_{0}[\theta, \lambda]$ has the form

$$
S_{0}[\theta, \lambda]=\frac{1}{\beta} \sum_{\omega}\left(\lambda^{T}(-\omega) B(\omega) \lambda(\omega)+i \lambda^{T}(-\omega) \theta(\omega)\right)
$$

with

$$
B(\omega)=\int_{-\infty}^{\infty} \frac{d k}{k^{2}} A^{-1}(\omega, k)=\oint \frac{\tilde{A}(\omega, k) d k}{k^{2} \operatorname{det} A(\omega, k)}
$$

where $\tilde{A}(\omega, k)$ is the adjugate matrix of $A(\omega, k)$. The matrix $B(\omega)$ can be explicitly evaluated. The determinant of $A(\omega, k)$ is given by $\operatorname{det} A=g^{4}-2 g^{2}\left(a_{1} a_{2}-b_{1} b_{2}\right)+\left(a_{1}^{2}+b_{1}^{2}\right)\left(a_{2}^{2}+b_{2}^{2}\right)$ where $g=\sqrt{\left(v_{1}-v_{2}\right)^{2}+4 \xi}$ with $\xi=\nu_{1} \nu_{2} g_{12}^{2}$. It is equivalent to

$$
\nu_{1}^{2} \nu_{2}^{2} k^{4} \operatorname{det} A=\eta^{2}\left(k^{2}+k_{+}^{2}\right)\left(k^{2}+k_{-}^{2}\right)
$$

where $\eta=v_{1} v_{2}-\xi . k_{ \pm}$are positive constants which satisfy

$$
k_{+}^{2}+k_{-}^{2}=\left(v_{1}^{2}+v_{2}^{2}+2 \xi\right) \omega^{2} / \eta^{2}, \quad k_{+}^{2} k_{-}^{2}=\omega^{2} /|\eta| .
$$

It turns out that finite components are $B_{11}=B_{22}, B_{33}=B_{44}$, and $B_{13}=B_{24}=B_{31}=B_{42}$. The other components vanish since their integrands have odd powers of $\mathrm{k}$. We obtain 


$$
B_{11}=\frac{\pi \nu_{1}}{\eta^{2}} \frac{v_{1}|\eta|+v_{2} \eta}{k_{+}+k_{-}}, \quad B_{33}=\frac{\pi \nu_{2}}{\eta^{2}} \frac{v_{1} \eta+v_{2}|\eta|}{k_{+}+k_{-}}, \quad B_{13}=\frac{\pi g \nu_{1} \nu_{2}}{\eta^{2}} \frac{|\eta|-\eta}{k_{+}+k_{-}}
$$

where $p=\left(v_{1}-v_{2}\right) / g, q=2 \nu_{1} \nu_{2} g_{12} / g$. Rearranging the fields as $\lambda^{( \pm) T}=\left(\lambda_{1}^{( \pm)}, \lambda_{2}^{( \pm)}\right), \theta^{( \pm) T}=$ $\left(\theta_{1}^{( \pm)}, \theta_{2}^{( \pm)}\right)$, we can write the action (9) in the form $S_{0}[\theta, \lambda]=S_{0}^{(+)}\left[\theta^{(+)}, \lambda^{(+)}\right]+S_{0}^{(-)}\left[\theta^{(-)}, \lambda^{(-)}\right]$, where

$$
S_{0}^{( \pm)}\left[\theta^{( \pm)}, \lambda^{( \pm)}\right]=\frac{1}{\beta} \sum_{\omega}\left(\frac{\pi}{|\omega|} \lambda^{( \pm) T}(-\omega) P \lambda^{( \pm)}(\omega)+i \lambda^{ \pm T}(-\omega) \theta^{ \pm}(\omega)\right)
$$

The matrix $\mathrm{P}$ is given by

$$
P=\left\{\begin{array}{r}
\frac{v_{1}+v_{2}}{\left|v_{1}+v_{2}\right|}\left[\begin{array}{rr}
\nu_{1} & 0 \\
0 & \nu_{2}
\end{array}\right] \quad(\eta>0) \\
{\left[\begin{array}{rr}
\nu_{1} p & q \\
q & -\nu_{2} p
\end{array}\right] \quad(\eta<0)}
\end{array}\right.
$$

Integrating out $\lambda$ 's, we finally obtain the effective action for $\theta$ as $S_{0}[\theta]=S_{0}^{(+)}\left[\theta^{(+)}\right]+S_{0}^{(-)}\left[\theta^{(-)}\right]$ with

$$
S_{0}^{( \pm)}\left[\theta^{( \pm)}\right]=\frac{1}{4 \pi \beta} \sum_{\omega}|\omega| \theta^{( \pm) T}(-\omega) P^{-1} \theta^{( \pm)}(\omega)
$$

The partition function of the system now reduces to $Z=\int \mathcal{D} \theta e^{-S_{0}[\theta]-\int d \tau U[\theta(\tau)]}$. Note that $\theta^{(+)}$ and $\theta^{(-)}$are decoupled in $S_{0}$.

\section{B. Stability condition}

In later sections, we will show that the scaling dimensions of the tunneling amplitudes are universal if both of the channels propagate in the same direction. The stability of the system is crucial for this universality.

The stability of the system requires that the Hamiltonian should be positive definite. In order to proceed it is convenient to transform the action into a representation in which both $K$ and $v$ are diagonal. This can be accomplished by performing the following transformations 23,24: $\phi=\Lambda \tilde{\phi}=\Lambda_{1} \Lambda_{2} \Lambda_{3} \tilde{\phi}$, where the first step is the diagonalization of the $K$ matrix: $\left(\Lambda_{1}^{T} K \Lambda_{1}\right)_{I J}=\delta_{I J} / \nu_{I}$ with $\left(\Lambda_{1}^{T} \Lambda_{1}\right)_{I J}=\delta_{I J}$. The second step is given by $\left(\Lambda_{2}\right)_{I J}=\sqrt{\left|\nu_{I}\right|} \delta_{I J}$ and $\Lambda_{2}^{T} \Lambda_{1}^{T} K \Lambda_{1} \Lambda_{2}=\Sigma$ where $\Sigma_{I J}=\sigma_{I} \delta_{I J}$ with $\sigma_{I}=\nu_{I} /\left|\nu_{I}\right|$. In the final step we choose as $\Lambda_{3}^{T} \Sigma \Lambda_{3}=\Sigma$ and $\Lambda_{3}^{T} \Lambda_{2}^{T} \Lambda_{1}^{T} v \Lambda_{1} \Lambda_{2} \Lambda_{3}=\tilde{v}$ with $\tilde{v}=\tilde{v}_{I} \delta_{I J}$. In the $\tilde{\phi}$-representation the action $(2)$ has the form in which all the channels are decoupled,

$$
S_{0}=\sum_{I} \int d \tau d x\left[\frac{1}{8 \pi} \tilde{v}_{I}\left\{\left(\frac{\partial \phi_{I}^{(+)}}{\partial x}\right)^{2}+\left(\frac{\partial \phi_{I}^{(-)}}{\partial x}\right)^{2}\right\}+\frac{i}{4 \pi} \sigma_{I} \frac{\partial \phi_{I}^{(+)}}{\partial \tau} \frac{\partial \phi_{J}^{(-)}}{\partial x}\right] .
$$

The situation is similar for the Hamiltonian

$$
\begin{aligned}
H & =H^{(u)}+H^{(l)}=\frac{1}{8 \pi} \int d x \sum_{I, J} v_{I J}\left(\rho_{I}^{(u)}(x) \rho_{J}^{(u)}(x)+\rho_{I}^{(l)}(x) \rho_{J}^{(l)}(x)\right) \\
& =\frac{1}{8 \pi} \int d x \sum_{I} \tilde{v}_{I}\left\{\left(\tilde{\rho}_{I}^{(u)}(x)\right)^{2}+\left(\tilde{\rho}_{I}^{(l)}(x)\right)^{2}\right\}
\end{aligned}
$$


where $\rho_{I}^{(u, l)}(x)=J_{0 I}^{(u, l)}(x)=\partial_{1} \phi_{I}^{(u, l)}(x) / 2 \pi$, and $\tilde{\rho}_{I}^{(u, l)}(x)=\tilde{J}_{0 I}^{(u, l)}(x)=\partial_{1} \tilde{\phi}_{I}^{(u, l)}(x) / 2 \pi$ satisfy the following commutation relations,

$$
\left[\rho_{I}^{(u, l)}(k), \rho_{J}^{(u, l)}(-k)\right]= \pm \frac{k}{2 \pi}\left(K^{-1}\right)_{I J}, \quad\left[\tilde{\rho}_{I}^{(u, l)}(k), \tilde{\rho}_{J}^{(u, l)}(-k)\right]= \pm \frac{k}{2 \pi} \sigma_{I} \delta_{I J}
$$

The stability of the system requires that all $\tilde{v}_{I}$ 's should be positive. In our two-channel model, $K$ and $v$ are given in (3). Since $K$ has been already been diagonalized, $\Lambda_{1}$ is chosen to be an identity. The choice of $\Lambda_{2}$ is trivial. Finally we must choose $\Lambda_{3}$ such that $\Lambda_{3}^{T} \Sigma \Lambda_{3}=\Sigma$. Hence it is enough to study the following two cases [5].

(i) $\nu_{1} \nu_{2}>0$

In this case, the matrix $\Sigma$ is the identity except its sign, $\Sigma=\sigma I$ where $\sigma=\sigma_{1}=\sigma_{2}$. The matrix $\Lambda_{3}$ can be chosen as $\Lambda_{3}=\left[\begin{array}{rr}\cos \theta & \sin \theta \\ -\sin \theta & \cos \theta\end{array}\right]$ where $\theta$ must satisfy $\tan 2 \theta=-2 \sigma \sqrt{\xi} /\left(v_{1}-v_{2}\right)$. It can be shown that $\tilde{v}_{1}$ and $\tilde{v}_{2}$ are two different solutions of the quadratic equation $\tilde{v}^{2}-\sigma\left(v_{1}+v_{2}\right) \tilde{v}+\eta=$ 0 . Note that the discriminant $D=g^{2}$ is always positive, which ensure the real solutions for $v_{1}$ and $v_{2}$. Hence the stability condition reduces to $\sigma\left(v_{1}+v_{2}\right)>0$ and $\eta>0$. In this case, the matrix $P$ given in (11) becomes diagonal and has only positive universal components. Recall that $\eta=v_{1} v_{2}-\xi$ with $\xi=\nu_{1} \nu_{2} g_{12}^{2}$. In the present case since both $\xi$ and $\eta$ are positive, $v_{1} v_{2}>0$. Hence both channels propagate in the same direction.

(ii) $\nu_{1} \nu_{2}<0$

In this case, the matrix $\Lambda_{3}$ which satisfies $\Lambda_{3}^{T} \Sigma \Lambda_{3}=\Sigma$ has the form $\Lambda_{3}=\left[\begin{array}{cc}\cosh \theta & \sinh \theta \\ \sinh \theta & \cosh \theta\end{array}\right]$ where $\tanh 2 \theta=-2 \sigma_{1} \sqrt{-\xi} /\left(v_{1}-v_{2}\right)$. It is shown that $\tilde{v}_{1}$ and $\tilde{v}_{2}$ satisfy the following quadratic equation respectively: $\tilde{v}_{1,2}^{2}+\sigma_{1,2}\left(v_{1}+v_{2}\right) \tilde{v}_{1,2}+\eta=0$. The requirement of stability is that both $\tilde{v}_{1}$ and $\tilde{v}_{2}$ should have a positive solution, which is equivalent to $\eta<0$. The matrix $P$ is non-universal and has off-diagonal components. In the present case since both $\xi$ and $\eta$ are negative, $v_{1} v_{2}<0$. Hence both channels propagate in the opposite direction.

\section{QUASIPARTICLE TUNNELING PICTURE}

In this section, we first consider the limit where the scattering potential is very weak, i.e. we start the RG analysis from the situation where the upper and lower branches are separated by the bulk FQH liquid. Quasiparticle tunnelings are possible only near the point contact since only there not only upper and lower branches approach but also the translational symmetry breaks down.

Performing the RG transformation, we calculate exactly the scaling dimensions of the scattering amplitudes. Next we consider the opposite limit where the scattering potential is very strong. In this limit, the electron transport can be viewed as the tunneling of the phase $\theta$ from a potential minimum to an adjacent minimum. This process corresponds to an instanton or an anti-instanton. By the duality mapping we transform the original model to an analogous model in the weak potential limit and RG analysis of the tunneling amplitude is given.

It will be shown that the scaling dimensions are universal when $\nu_{1} \nu_{2}>0$, while it depends on the interaction when $\nu_{1} \nu_{2}<0$. In case (i) most of the processes are relevant, but for the $(1,-1 / 3)$ state only $\alpha_{22}$ is positive, which results in a different situation for the tunneling at low temperature. 


\section{A. Quasiparticle tunneling at a point contact}

Now we analyze in detail the backward scatterings at a point contact. The scattering potential at $x=0$ has the form $U=U_{11}+U_{22}+U_{12}$ where $U_{I I}$ corresponds to the intra-channel quasiparticle tunneling process:

$$
U_{I I}=-u_{I I} \cos \theta_{I}^{(+)}(\tau) \propto\left(\Psi_{I}^{(u) \dagger} \Psi_{I}^{(l)}\right)^{\nu_{I}}+\text { h.c. }
$$

where $\Psi_{I}^{(u, l)}$ is the electron operator on the upper (lower) edge. In terms of the bose field $\theta_{I}^{(u, l)}$ the electron operator can be written as

$$
\Psi_{I}^{(u, l)}(\tau)=\sqrt{\bar{\rho}} \exp \left( \pm i \theta_{I}^{(u, l)}(\tau) / \nu_{I}\right)
$$

A similar formula is given in (A5) in the appendix. The other term, which will be proven to be important for our following discussions, describes inter-channel scatterings. For $\nu_{1} \nu_{2}>0$, it has the form $U_{12}=U_{12}^{u l}+U_{12}^{l u}$ where

$$
\begin{aligned}
& U_{12}^{u l}=-u_{12}^{u l} \cos \frac{\theta_{+}^{(+)}(\tau)+\theta_{-}^{(-)}(\tau)}{2} \propto\left(\Psi_{1}^{(u)} \Psi_{2}^{(l) \dagger}\right)^{\nu_{1}}+\text { h.c. }, \\
& U_{12}^{l u}=-u_{12}^{l u} \cos \frac{\theta_{+}^{(+)}(\tau)-\theta_{-}^{(-)}(\tau)}{2} \propto\left(\Psi_{1}^{(l)} \Psi_{2}^{(u) \dagger}\right)^{\nu_{1}}+\text { h.c. }
\end{aligned}
$$

For $\nu_{1} \nu_{2}<0, U_{12}=U_{12}^{u u}+U_{12}^{l l}$ where

$$
\begin{aligned}
& U_{12}^{u u}=-u_{12}^{u u} \cos \frac{\theta_{+}^{(+)}(\tau)+\theta_{+}^{(-)}(\tau)}{2} \propto\left(\Psi_{1}^{(u)} \Psi_{2}^{(u)}\right)^{\nu_{1}}+\text { h.c. }, \\
& U_{12}^{l l}=-u_{12}^{l l} \cos \frac{\theta_{+}^{(+)}(\tau)-\theta_{+}^{(-)}(\tau)}{2} \propto\left(\Psi_{1}^{(l)} \Psi_{2}^{(l)}\right)^{\nu_{1}}+\text { h.c. }
\end{aligned}
$$

Here $\theta_{ \pm}^{( \pm)}=\theta_{1}^{( \pm)} \pm \rho \theta_{2}^{( \pm)}$and $\rho=\nu_{1} / \nu_{2}$, and we assumed that $\left|\nu_{1}\right|>\left|\nu_{2}\right|$ with $\rho$ being an odd integer. $U_{12}$ corresponds to inter-branch (intra-branch) tunnelings for $\nu_{1} \nu_{2}>0\left(\nu_{1} \nu_{2}<0\right)$. In the following we also assume $u_{12}^{u l}=u_{12}^{l u}=u_{12}$ for $\nu_{1} \nu_{2}>0$ and $u_{12}^{u u}=u_{12}^{l l}=u_{12}$ for $\nu_{1} \nu_{2}<0$. Hence for $\nu_{1} \nu_{2}>0$

$$
U_{12}=-2 u_{12} \cos \frac{\theta_{+}^{(+)}(\tau)}{2} \cos \frac{\theta_{-}^{(-)}(\tau)}{2}
$$

and for $\nu_{1} \nu_{2}<0$

$$
U_{12}=-2 u_{12} \cos \frac{\theta_{+}^{(+)}(\tau)}{2} \cos \frac{\theta_{+}^{(-)}(\tau)}{2} .
$$

We assume that all $u_{I J}$ 's are positive but the result does not depend on the signs of $u_{I J}$ 's.

\section{B. Weak potential limit}

We first consider the limit where the scattering potential is very weak. We calculate the scaling dimensions of the scattering amplitudes by recursively integrating out the highfrequency modes [2] [3] [25]. At zero temperature, the partition function can be written as $Z=\int \mathcal{D} \theta_{\Lambda} e^{-S_{0}\left[\theta_{\Lambda}\right]-\int d \tau U_{\Lambda}\left[\theta_{\Lambda}(\tau)\right]}$. Here we introduced a cutoff $\Lambda$ such that 


$$
\theta_{\Lambda}(\omega)= \begin{cases}\theta(\omega) & (|\omega|<\Lambda) \\ 0 & (\Lambda<|\omega|)\end{cases}
$$

The unperturbed action $S_{0}\left[\theta_{\Lambda}\right]$ has the form $S_{0}\left[\theta_{\Lambda}\right]=S_{0}^{(+)}\left[\theta_{\Lambda}^{(+)}\right]+S_{0}^{(-)}\left[\theta_{\Lambda}^{(-)}\right]$where

$$
S_{0}^{( \pm)}\left[\theta_{\Lambda}^{( \pm)}\right]=\frac{1}{4 \pi \beta} \sum_{\omega}|\omega| \theta_{\Lambda}^{( \pm) T}(-\omega) P^{-1} \theta_{\Lambda}^{( \pm)}(\omega)
$$

We divide the field $\theta_{\Lambda}(\tau)$ into slow and fast modes as $\theta_{\Lambda}(\tau)=\theta_{\mu}(\tau)+\theta_{\text {fast }}(\tau)$ such that

$$
\theta_{\Lambda}(\omega)= \begin{cases}\theta_{\text {fast }}(\omega) & (\mu<|\omega|<\Lambda) \\ \theta_{\mu}(\omega) & (|\omega|<\mu)\end{cases}
$$

Integrating out the fast modes, we obtain an effective action for the slow modes $\theta_{\mu}$ : $Z=$ $\int \mathcal{D} \theta_{\mu} e^{-S_{0}\left[\theta_{\mu}\right]-\int d \tau U(\mu)\left[\theta_{\mu}(\tau)\right]}$. Here we keep only the first order terms with respect to the scattering potential $\mathrm{U}$ since they are most relevant. We define the scaling dimension $\alpha_{I J}$ as

$$
\frac{u_{I J}(\mu)}{\mu}=\left(\frac{\mu}{\Lambda}\right)^{\alpha_{I J}} \frac{u_{I J}(\Lambda)}{\Lambda}
$$

for $I, J=1,2$. These are determined by the equations $u_{I I}(\mu)=e^{-\frac{1}{2} G_{I I}(0)} u_{I I}(\Lambda)$ for $I=1,2$, and $u_{12}(\mu)=e^{-\frac{1}{4}\left(G_{11}(0)+\rho^{2} G_{22}(0)-2 \rho G_{12}(0)\right)} u_{12}(\Lambda)$. $G_{I J}$ 's are the correlation functions of the fast modes:

$$
G_{I J}(\tau)=\left\langle\theta_{I f a s t}^{ \pm}(\tau) \theta_{J f a s t}^{ \pm}(0)\right\rangle=P_{I J} \int_{|\omega|<\Lambda} d \omega \frac{e^{-i \omega \tau}}{|\omega|} W\left(\frac{\mu}{\omega}\right)
$$

where $W(x)$ is a smoothing function with $W(x) \rightarrow 0$ for $|x| \ll 1$ and $W(x) \approx 1$ for $x \gg 1$ [25]. $\alpha_{I J}$ 's are universal only when $\nu_{1} \nu_{2}>0$ corresponding to the form of matrix $P$ given in (15). The tunneling conductance scales at a "high" temperature as

$$
G(T)=G(\infty)-c s t . \times T^{2 \min \left[\alpha_{I J}\right]}
$$

where $G(\infty)$ is the quantized Hall conductance. The exponent $\alpha_{I J}$ 's are listed in Table 1 , and explicit values are presented for $\nu=2 / 5$ and $2 / 3$ cases in Table 2.

\section{Strong potential limit}

Here we consider the limit where the scattering potential is very strong. In this limit, the electron transport can be viewed as the tunneling from a potential minimum to its adjacent one, and tunneling matrix elements, i.e. instanton fugacities are the expansion parameters. Using the dilute instanton gas approximation (DIGA), we transform the original model to its dual theory in the weak potential limit and apply to it the RG analysis in the preceding subsection. In determining the minima of the scattering potential $U[\theta]$ at $x=0$, only $\theta_{1}=\theta_{1}^{(+)}, \theta_{2}=\theta_{2}^{(+)}$, and $\theta_{3}=\theta_{+}^{(-)}\left(\theta_{3}=\theta_{-}^{(-)}\right)$for $\nu_{1} \nu_{2}>0\left(\nu_{1} \nu_{2}<0\right)$ are independent. After integrating out the other degree of freedom, the partition function has the form $Z=\int \mathcal{D} \theta_{1} \int \mathcal{D} \theta_{2} \int \mathcal{D} \theta_{3} e^{-S_{0}\left[\theta_{1}, \theta_{2}, \theta_{3}\right]-\int d \tau U\left[\theta_{1}, \theta_{2}, \theta_{3}\right]}$ where

$$
S_{0}\left[\theta_{1}, \theta_{2}, \theta_{3}\right]=\frac{1}{4 \pi \beta} \sum_{\omega}|\omega| \theta^{T}(-\omega) Q \theta(\omega)
$$


with $\theta^{T}=\left(\theta_{1}, \theta_{2}, \theta_{3}\right)$ and $\mathrm{Q}$ is a 3 by 3 matrix which has the form

$$
Q=\left(\begin{array}{cc}
P^{-1} & \overrightarrow{0} \\
\overrightarrow{0}^{T} & 1 / \nu_{3}
\end{array}\right)
$$

where $\nu_{3}$ is defined as

$$
\nu_{3}= \begin{cases}\left|\nu_{1}\right|+\rho^{2}\left|\nu_{2}\right| & (\eta>0) \\ \left(\nu_{1}-\rho^{2} \nu_{2}\right) p+2 \rho q & (\eta<0)\end{cases}
$$

The scattering potential (22-25) now has the form $U=U_{1}+U_{2}+U_{3}$ where

$$
\begin{aligned}
& U_{1}=-u_{1} \cos \theta_{1}(\tau), \\
& U_{2}=-u_{2} \cos \theta_{2}(\tau), \\
& U_{3}=-u_{3} \cos \frac{\theta_{1}(\tau)+\rho \theta_{2}(\tau)}{2} \cos \frac{\theta_{3}}{2} .
\end{aligned}
$$

Hence the scattering potential has minima at $\left(\theta_{1}, \theta_{2}, \theta_{3}\right)=2 \pi(l, m, n)$ where $l, m, n$ are integers which satisfy the condition that $l+m$ is (a)even for even $\mathrm{n}$ and (b) odd for odd $\mathrm{n}$. It follows that the minima of the potential form a double-layer lattice in the three-dimensional $\theta_{1} \theta_{2} \theta_{3^{-}}$ space. The typical layers of that lattice are the equi- $\theta_{3}$ planes with (a) $\theta_{3}=4 k \pi$ and (b) $\theta_{3}=2(2 k+1) \pi$.

Using the DIGA we show below that the partition function in the strong potential limit is mapped to an analogous one in the weak potential limit [26]. In contrast to the single-channel case, it turns out that the dual theory is not identical to the original one.

In the DIGA the field $\theta_{I}(\tau)$ can be written as $\theta_{I}(\tau)=\sum_{j=1}^{9} C_{I j} X_{j}(\tau)$ where $C_{I j}$ 's are listed in Table $3 . X_{j}$ 's are linear combinations of instantons $\left(e_{j k}=1\right)$ and anti-instantons $\left(e_{j k}=1\right)$ : $X_{j}(\tau)=\sum_{k=1}^{n_{j}} e_{j k} X\left(\tau-\tau_{j k}\right)$ where $e_{j k}=1$ and $\tau_{j k}$ are respectively the topological charge and the central imaginary time of the $\mathrm{k}$-th instanton in the $\mathrm{j}$-th species. $X(\tau)$ is a one-instanton solution at $\tau=0$ the width of which should be neglected compared to $\beta$ in the DIGA. The instanton or anti-instanton in $X_{j}(\tau)$ which we call those of the $j$-th species corresponds to a tunneling process from $\left(\theta_{1}, \theta_{2}, \theta_{3}\right)$ to $\left(\theta_{1}, \theta_{2}, \theta_{3}\right)+2 \pi e_{j k}\left(C_{1 j}, C_{2 j}, C_{3 j}\right)$. Here we took into account only the nine species listed in Table 3 since they are most likely to be relevant.

Tunneling of an instanton or an anti-instanton along the $\theta_{1}$-axis, $\theta_{2}$-axis, and $\theta_{3}$-axis can be interpreted physically as a pulse of 1-channel current, 2-channel current and the total charge density fluctuation respectively. The bosonization of current and density is given for example in the appendix, where they are expressed as $J_{I}=\partial_{0} \theta_{I}^{(+)} / 2 \pi$ and $\rho_{I}=\partial_{0} \theta_{I}^{(-)} / 2 \pi$.

We may write the Fourier transform of $\theta_{I}(\tau)$ as

$$
\theta_{I}(\omega)=\frac{2 \pi i}{\omega} \sum_{j=1}^{9} C_{I j} \sum_{k=1}^{n_{j}} e_{j k} e^{i \omega \tau_{j k}}
$$

where we used the approximation $d X / d \tau \approx 2 \pi \delta(\tau)$.

The partition function now has the form $Z=\sum e^{-S_{0}\left[\theta_{1}, \theta_{2}, \theta_{3}\right]}$ where $\theta_{I}$ 's satisfy (36). $\sum$ denotes a "summation" over instanton configurations, or explicitly,

$$
\sum=\prod_{j=1}^{9} \sum_{n_{j}=0}^{\infty} \frac{y_{j}^{n_{j}}}{n_{j} !} \sum_{\left\{e_{j k}\right\}} \prod_{k=1}^{n_{j}} \int_{0}^{\beta} d \tau_{j k}
$$

where $y_{j}$ is the instanton fugacity, i.e. the tunneling matrix elements from $\left(\theta_{1}, \theta_{2}, \theta_{3}\right)=(0,0,0)$ to $2 \pi e_{j k}\left(C_{1 j}, C_{2 j}, C_{3 j}\right)$. We consider the Gaussian integral over the" dual fields": 


$$
\int \mathcal{D} \tilde{\theta} \exp \left(-\frac{1}{4 \pi \beta} \sum_{\omega}|\omega| \tilde{\theta}^{T}(-\omega) Q^{-1} \tilde{\theta}(\omega)\right)
$$

where $\tilde{\theta}^{T}=\left(\tilde{\theta}_{1}, \tilde{\theta}_{2}, \tilde{\theta}_{3}\right)$ and insert it into the "summation" in the partition function. Next we perform the shift transformation $\tilde{\theta}(\omega) \rightarrow \tilde{\theta}(\omega)+(\omega /|\omega|) \theta(\omega)$ which cancels the original action $S_{0}\left[\theta_{1}, \theta_{2}, \theta_{3}\right]$. We can write the partition function in the form

$$
Z=\sum \int \mathcal{D} \tilde{\theta} \exp \left[-\tilde{S}_{0}[\tilde{\theta}]-i \sum_{j=1}^{9} \sum_{k=1}^{n_{j}} e_{j k} \sum_{I=1}^{3} C_{I j} \tilde{\theta}_{I}\left(\tau_{j k}\right)\right]
$$

where

$$
\tilde{S}_{0}[\tilde{\theta}]=\frac{1}{4 \pi \beta} \sum_{\omega}|\omega| \tilde{\theta}^{T}(-\omega) Q^{-1} \tilde{\theta}(\omega)
$$

Taking the "summation" over instanton configurations, we can finally write the partition function in terms of the dual action $\tilde{S}[\tilde{\theta}]$ as $Z=\int \mathcal{D} \tilde{\theta} e^{-\tilde{S}[\tilde{\theta}]}$ where $\tilde{S}[\tilde{\theta}]=\tilde{S}_{0}[\tilde{\theta}]+\int d \tau Y[\tilde{\theta}(\tau)]$ with "the scattering potential"

$$
Y[\tilde{\theta}]=\sum_{j=1}^{9} 2 y_{j} \cos \left(\sum_{I=1}^{3} C_{I j} \tilde{\theta}_{I}\right)
$$

It turns out that the unperturbed part $\tilde{S}_{0}[\tilde{\theta}]$ of the dual action can be identified with the original $S_{0}[\theta]$ by the correspondences $Q^{-1} \leftrightarrow Q$ and $\tilde{\theta} \leftrightarrow \theta$. In contrast to the single-channel case, the potential term $Y[\tilde{\theta}]$ is analogous but not identical to the original potential $U[\theta]$ in spite of the correspondence $2 y_{j} \leftrightarrow-t_{I}$.

It is straight-forward to calculate the scaling dimensions $\beta_{j}$ of the tunneling matrix element $y_{j}$, which is defined by

$$
\frac{y_{j}(\mu)}{\mu}=\left(\frac{\mu}{\Lambda}\right)^{\beta_{j}} \frac{y_{j}(\Lambda)}{\Lambda}
$$

These are calculated using the equations

$$
\begin{aligned}
y_{1}(\mu) & =e^{-\frac{1}{2}\left(\tilde{G}_{11}(0)+\tilde{G}_{22}(0)+2 \tilde{G}_{12}(0)\right)} y_{1}(\Lambda), \\
y_{2}(\mu) & =e^{-\frac{1}{2}\left(\tilde{G}_{11}(0)+\tilde{G}_{22}(0)-2 \tilde{G}_{12}(0)\right)} y_{2}(\Lambda), \\
y_{3,4}(\mu) & =e^{-\frac{1}{2}\left(\tilde{G}_{22}(0)+\tilde{G}_{33}(0)\right)} y_{3,4}(\Lambda), \\
y_{5,6}(\mu) & =e^{-\frac{1}{2}\left(\tilde{G}_{33}(0)+\tilde{G}_{11}(0)\right)} y_{5,6}(\Lambda), \\
y_{7,8,9}(\mu) & =e^{-2 \tilde{G}_{11,22,33}(0)} y_{7,8,9}(\Lambda),
\end{aligned}
$$

where $\tilde{G}_{I J}$ 's are the correlation functions of the fast modes:

$$
\tilde{G}_{I J}(\tau)=\left\langle\tilde{\theta}_{I f a s t}^{ \pm}(\tau) \tilde{\theta}_{J f a s t}^{ \pm}(0)\right\rangle=Q_{I J} \int_{|\omega|<\Lambda} d \omega \frac{e^{-i \omega \tau}}{|\omega|} W\left(\frac{\mu}{\omega}\right) .
$$

The results are listed in Table 4 . The explicit values of $\beta_{j}$ for some specific edge constructions are shown in Table 5 . In the following we give a detailed discussion on the temperature dependence of the tunneling conductance in the dilute instanton gas (DIG) picture. 


\section{DIG picture for $\nu_{1} \nu_{2}>0$}

First we consider the case where both of the edge channels propagate in the same direction. In Sec. II we have shown that $\alpha_{I J}$ 's are universal only for $\nu_{1} \nu_{2}>0$, which is also the case with $\beta_{j}$ 's (Table 4).

Table 5 of $\beta_{j}$ 's tells us that all instanton fugacities scale to zero when $\nu_{1} \nu_{2}>0$ (i). In this case we can determine the temperature dependence of the tunneling conductance using DIG picture: $G(T) \propto T^{\delta_{D I G}}$ where $\delta_{D I G}=2 \min \left[\beta_{j}\right]$. It should also be noted that the inter-channel tunnelings play a crucial role in determining $\delta_{D I G}$. In Table 6 the explicit values of $\delta_{D I G}$ is shown, for comparison, in the presence and in the absence of inter-channel tunnelings.

\section{DIG picture for $\left(\nu_{1}, \nu_{2}\right)=(1,-1 / 3)$}

Here we consider the $(1,-1 / 3)$ state which belongs to case (ii): $\nu_{1} \nu_{2}<0$. As was shown in Table 4 the exponents depend on the interaction between the channels, and not all of the instanton fugacities scale to zero, i.e. the one $\left(y_{9}\right)$ which corresponds to $\theta_{3}=\theta_{-}^{(-)}$scales to a larger value. In Sec. II we have shown that the scattering potential $U_{12}$ which suppress the fluctuation of $\theta_{3}=\theta_{-}^{(-)}$is irrelevant. Hence at both strong and weak potential limit $\theta^{(-)}$is not pinned.

In contrast to the neutral mode $\theta_{3}=\theta_{-}^{(-)}$all $y_{j}$ 's which describe the instanton tunneling in the $\theta_{1}^{(+)}$or $\theta_{2}^{(+)}$direction scale to zero, i.e. both $\theta_{1}^{(+)}$and $\theta_{2}^{(+)}$are fixed. However it is shown in Sec. II that only $\theta_{2}^{(+)}$is pinned at weak potential limit. These two pictures in the strong and weak potential limits are not smoothly connected. They can be understood by considering a RG flow diagram of the type shown in Fig. 1, which was constructed in the following way.

The inter-channel tunnelings are irrelevant at both of the strong and weak potential limit for the $(1,-1 / 3)$ state and can be neglected. It is enough to study only the intra-channel tunnelings. In the following we study three DIG pictures.

First we consider the situation where only $\theta_{1}^{(+)}$is pinned. We start with the action

$$
S=S_{0}^{(+)}\left[\theta^{(+)}\right]+\int_{0}^{\beta} d \tau\left(U_{1}\left[\theta_{1}^{(+)}\right]+U_{2}\left[\theta_{2}^{(+)}\right]\right)
$$

where $S_{0}^{(+)}$was given in (16), and $U_{1}$ and $U_{2}$ were introduced in (35). The scaling dimension of $u_{2}$ can be calculated as

$$
\frac{u_{2}(\mu)}{\mu}=\left(\frac{\mu}{\Lambda}\right)^{-\nu_{2} p-1} \frac{u_{2}(\Lambda)}{\Lambda}
$$

irrespective of the strength of $u_{1}$. The scattering potential $u_{2}$ is considered to be relevant also in the DIG picture. On the other hand on the axis $u_{2}=0$ the effective action for $\theta_{1}^{(+)}$is obtained by integrating out $\theta_{2}^{(+)}$:

$$
S=\frac{1}{4 \pi \beta} \sum_{\omega}|\omega| \theta_{1}^{(+)}(-\omega) \frac{1}{\nu_{1} p} \theta_{1}^{(+)}(\omega)-u_{1} \int_{0}^{\beta} \cos \theta_{1}^{(+)}(\tau)
$$

where we used the relation $p=\left(v_{1}-v_{2}\right) / g, q=2 \nu_{1} \nu_{2} g_{12} / g, g=\sqrt{\left(v_{1}-v_{2}\right)^{2}+4 \xi}$ and $\xi=$ $\nu_{1} \nu_{2} g_{12}^{2}$. We can apply DIGA to the action (47) to obtain its dual version

$$
\tilde{S}=\frac{1}{4 \pi \beta} \sum_{\omega}|\omega| \tilde{\theta}_{1}^{(+)}(-\omega) \nu_{1} p \tilde{\theta}_{1}^{(+)}(\omega)-z_{1} \int_{0}^{\beta} \cos \tilde{\theta}_{1}^{(+)}(\tau)
$$


where $z_{1}$ is the instanton fugacity, the scaling dimension of which is obtained as

$$
\frac{z_{1}(\mu)}{\mu}=\left(\frac{\mu}{\Lambda}\right)^{1 / \nu_{1} p-1} \frac{z_{1}(\Lambda)}{\Lambda}
$$

Since $1 / \nu_{1} p-1<0$ for the $(1,-1 / 3)$ state the instanton fugacity $z_{1}$ is relevant. In terms of the original scattering potential $u_{1}$ it tends to decrease in the large- $u_{1}$ region. Recall that $u_{1}$ is irrelevant in the small- $u_{1}$ region. We conclude that both the original and the dual picture belong to the domain (a) of Fig. 1.

Next we consider the situation where only $\theta_{2}^{(+)}$is pinned. In this case the same procedure gives us

$$
\frac{u_{1}(\mu)}{\mu}=\left(\frac{\mu}{\Lambda}\right)^{\nu_{1} p-1} \frac{u_{1}(\Lambda)}{\Lambda}, \quad \frac{z_{2}(\mu)}{\mu}=\left(\frac{\mu}{\Lambda}\right)^{-1 / \nu_{2} p-1} \frac{z_{2}(\Lambda)}{\Lambda}
$$

where $-1 / \nu_{2} p-1=3 / p-1>0$. It turns out that the scattering potential $u_{1}$ is irrelevant but $u_{2}$ tends to increase, which indicates that $(0, \infty)$ is an atractive fixed point in the $\left(u_{1}, u_{2}\right)$-plane.

Finally we consider the case where both $\theta_{1}^{(+)}$and $\theta_{2}^{(+)}$are pinned. We start with the action (45) and perform the duality mapping to obtain

$$
\tilde{S}=\frac{1}{4 \pi \beta} \sum_{\omega}|\omega| \tilde{\theta}^{(+)}(-\omega) P \tilde{\theta}^{(+)}(\omega)-\sum_{J=1,2} z_{J} \int_{0}^{\beta} \cos \tilde{\theta}_{J}^{(+)}(\tau)
$$

where $\mathrm{P}$ is a $2 \times 2$ matrix given in (15). The scaling dimensions of $z_{1}$ and $z_{2}$ are calculated as

$$
\frac{z_{1}(\mu)}{\mu}=\left(\frac{\mu}{\Lambda}\right)^{p / \nu_{1}-1} \frac{z_{1}(\Lambda)}{\Lambda}, \quad \frac{z_{2}(\mu)}{\mu}=\left(\frac{\mu}{\Lambda}\right)^{-p / \nu_{2}-1} \frac{z_{2}(\Lambda)}{\Lambda}
$$

where we used the relation $\operatorname{det} P=-\nu_{1} \nu_{2}$. It turns out that both of the instanton fugacities are irrelevant. In terms of the original scattering potential, both $u_{1}$ and $u_{2}$ tend to increase, i.e. $(\infty, \infty)$ is another atractive fixed point in the $\left(u_{1}, u_{2}\right)$-plane. It is evident that this picture is not smoothly connected to the above two DIG pictures where only one of the scattering potential is strong. Therefore we expect that there are two domains in the RG flow diagram in terms of the original scattering potential $u_{1}$ and $u_{2} .(0, \infty)$ and $(\infty, \infty)$ are the atractive fixed points in the domain (a) and (b) of Fig. 1, respectively.

\section{ELECTRON TUNNELING PICTURE}

In the latter half of the preceding section, we consider the limit of strong scattering potential in terms of duality mapping. There it was revealed that the tunneling amplitude through the potential is irrelevant in most of the cases. Then it would be more appropriate to start with the picture where the bulk FQH state is divided into left and right condensates and electrons tunnel between the left and right edges through a insulating region at the point contact.

First we study the electron tunneling (ET) picture for $\nu_{1} \nu_{2}>0$. As was pointed out before, the situation is different for the $(1,-1 / 3)$ state. In this case we must consider two different ET pictures both of which correspond to two atractive fixed points in the $\left(u_{1}, u_{2}\right)$-plane, respectively. These subjects will be discussed toward the end of this section. 


\section{A. Construction of the general electron operator}

At a point contact, two channels are close to each other within the magnetic length, the singlechannel electron operator $\Psi_{I}^{(L, R)}$ on the left (right) branch is no longer the most general one. The general electron operator $\Psi^{(L, R)}$ may contain charge transfers between the edges of the same branch and takes the following form [5]

$$
\Psi^{(L, R)}(s=0)=\sum_{n=-\infty}^{\infty} c_{n} \eta^{(L, R) n} \Psi_{1}^{(L, R)}(x=0)
$$

where the elementary charge transfer $\eta^{(L, R)}$ is given by

$$
\eta^{(L, R)}=\left(\Psi_{2}^{(L, R)} \Psi_{1}^{(L, R) \dagger}\right)^{\nu_{1}}=\exp \left[ \pm i\left(\phi_{1}^{(L, R)}(x=0)-\rho \phi_{2}^{(L, R)}(x=0)\right)\right]
$$

The electron tunneling from the left to right edge is expressed by the operator $\Gamma=$ $\left.\gamma \Psi^{(L)} \Psi^{(R) \dagger}\right|_{x=0}$.

\section{B. ET picture for $\nu_{1} \nu_{2}>0$}

First we consider the case where the two channels propagate in the same direction. The electron tunneling through a point contact can be described using the concept of tunneling Hamiltonian [27 $H_{T}=\Gamma+\Gamma^{\dagger}$ where field operators on the left and right edges anticommute, which in the present picture corresponds to the fact that two edges are those of independent incompressible fluids. We consider a nonequilibrium situation where the chemical potentials on each of the edges are different by the applied voltage: $\mathrm{eV}=\mu_{L}-\mu_{R}$.

The tunneling current $I(t)$ from the left to right edge is given by the average value of the operator $e d N_{L} / d t$ where $N_{L}$ is the number of particles on the left edge. Since we concentrate on the linear response of $I(t)$ with respect to $V$, we can evaluate the average using Kubo formula: $I(t)=i e \int_{-\infty}^{t} d t^{\prime}\left\langle\left[d N_{L}(t) / d t, H_{T}\left(t^{\prime}\right)\right]\right\rangle$ where $d N_{L}(t) / d t=i\left(\Gamma(t)-\Gamma^{\dagger}(t)\right)$. In terms of the Fourier transform of the retarded Green's function $X_{\text {ret }}(\tau)=-i \theta(\tau)\left\langle\left[\Gamma(\tau), \Gamma^{\dagger}(0)\right]\right\rangle$ we can write the current as $I(t)=2 e \Im\left[X_{\text {ret }}(\omega=-e V)\right]$. Hence the scaling dimension $\delta_{E T}$ of the tunneling conductance in the electron tunneling picture is equal to that of $|\gamma|^{2}$ [4], 5 ]:

$$
\frac{\gamma(\mu)}{\mu}=\left(\frac{\mu}{\Lambda}\right)^{\delta_{E T} / 2} \frac{\gamma(\Lambda)}{\Lambda}
$$

We can determine the scaling dimension of $\gamma$ by applying the RG analysis to the partition function, which can be written in terms of $\theta^{(L, R)}(\tau)=\phi^{(L, R)}(\tau, x=0)$ as

$$
Z=\int \mathcal{D} \theta e^{-S_{0}^{(L)}\left[\theta^{(L)}\right]-S_{0}^{(R)}\left[\theta^{(R)}\right]-\int d \tau H_{T}\left[\theta^{(L)}(\tau), \theta^{(R)}(\tau)\right]}
$$

where

$$
S_{0}^{(L, R)}\left[\theta^{(L, R)}\right]=\frac{1}{4 \pi \beta} \sum_{\omega}|\omega| \theta^{(L, R) T}(-\omega)\left[\begin{array}{cc}
2 /\left|\nu_{1}\right| & 0 \\
0 & 2 /\left|\nu_{2}\right|
\end{array}\right] \theta^{(L, R)}(\omega) .
$$

with $\theta^{(L, R) T}=\left(\theta_{1}^{(L, R)}, \theta_{2}^{(L, R)}\right)$. Here we have integrated out the fields except at the point contact. In the present picture $\theta^{(L)}$ and $\theta^{(R)}$ are not canonically conjugate but are the independent degrees of freedom. 
Next we introduce a cutoff $\Lambda$ and apply the RG analysis in the preceding section. Performing the integral over the fast modes, we obtain

$$
\gamma(\mu)=\gamma(\Lambda) \prod_{L, R} \max \left[e^{-\frac{1}{2}\left\{\left(1 /\left|\nu_{1}\right|-n\right)^{2} \hat{G}_{11}(0)+n^{2} \rho^{2} \hat{G}_{22}(0)\right\}}\right]
$$

where $\hat{G}_{I J}$ is

$$
\hat{G}_{I J}(\tau)=\left\langle\theta_{I f a s t}^{(L, R)}(\tau) \theta_{J f a s t}^{(L, R)}(0)\right\rangle=\frac{\left|\nu_{I}\right|}{2} \delta_{I J} \int_{|\omega|<\Lambda} d \omega \frac{e^{-i \omega \tau}}{|\omega|} W\left(\frac{\mu}{\omega}\right)
$$

Using $\nu_{3}=\left|\nu_{1}\right|+\rho^{2}\left|\nu_{2}\right|>0$ we can write $\delta_{E T}$ as

$$
\delta_{E T}=2\left(\frac{1}{\left|\nu_{1}\right|}+\nu_{3} \min \left[\left(n-\frac{1}{\nu_{3}}\right)^{2}\right]-\frac{1}{\nu_{3}}\right)-2 .
$$

It is shown that the minimum value is obtained when $n=1$ for $\left(\nu_{1}, \nu_{2}\right)=(1 / 3,1 / 15), n=1$ or 2 for $\left(\nu_{1}, \nu_{2}\right)=(1 / 3,1 / 3)$ and $n=2$ or 3 for $\left(\nu_{1}, \nu_{2}\right)=(1 / 5,1 / 5)$, respectively. Hence $\delta_{E T}$ 's are evaluated as

$$
\delta_{E T}=\left\{\begin{array}{lll}
4 & \text { for } & \left(\nu_{1}, \nu_{2}\right)=(1 / 3,1 / 15) \\
4 / 3 & \text { for } & \left(\nu_{1}, \nu_{2}\right)=(1 / 3,1 / 3) \\
16 / 5 & \text { for } & \left(\nu_{1}, \nu_{2}\right)=(1 / 5,1 / 5)
\end{array} .\right.
$$

\section{ET picture for $\left(\nu_{1}, \nu_{2}\right)=(1,-1 / 3)$}

For the $(1,-1 / 3)$ state we must consider two different ET pictures. In Sec. III we have shown that there are two attractive fixed points in the $\left(u_{1}, u_{2}\right)$-plane, $(0, \infty)$ and $(\infty, \infty)$. We can consider the ET picture in the vicinity of these points.

In Sec. III we performed the RG analysis near $\left(u_{1}, u_{2}\right)=(0,0)$, where we discuss the interchannel tunneling process $U_{12}$ as well as $U_{11}$ and $U_{22}$. Here we concentrate on the analysis of intra-channel tunnelings $U_{1}=U_{11}$ and $U_{2}=U_{22}$ since the RG flow of $\theta^{(-)}$have turned out to be trivial.

For $\left(u_{1}, u_{2}\right)=(0, \infty)$ we start with the situation where only the 2 -channel is reflected. In this case we slightly modify the discussion in Sec. III as

$$
U_{2} \rightarrow \Gamma_{2}=\gamma_{2} \cos \frac{\theta_{2}^{(+)}}{\nu_{2}}
$$

since we consider the electron tunneling from the left to right branch for the 2-channel. We obtain

$$
\frac{\gamma_{2}(\mu)}{\mu}=\left(\frac{\mu}{\Lambda}\right)^{-p / \nu_{2}-1} \frac{\gamma_{2}(\Lambda)}{\Lambda}
$$

where $-p / \nu_{2}-1=3 p-1 \rightarrow 2$ as $g_{12} \rightarrow 0$. We confirm that $(0, \infty)$ is a stable fixed point. Recall that in the DIG picture the scaling dimension of instanton fugacity $z_{1}$ in the present region is given by $-1 / \nu_{2} p-1=3 / p-1$ which is smaller than $-p / \nu_{2}-1=3 p-1$ since $p>1$. We predict the existence of a crossover between the two pictures. 
For $\left(u_{1}, u_{2}\right)=(\infty, \infty)$ we start the RG analysis with the picture where both of the channels are reflected: $U_{1} \rightarrow \Gamma_{1}=\gamma_{1} \cos \left(\theta_{1}^{(+)} / \nu_{1}\right), U_{2} \rightarrow \Gamma_{2}=\gamma_{2} \cos \left(\theta_{2}^{(+)} / \nu_{2}\right)$. We obtain $\gamma_{1}(\mu) / \mu=$ $(\mu / \Lambda)^{p / \nu_{1}-1} \gamma_{1}(\Lambda) / \Lambda, \gamma_{2}(\mu) / \mu=(\mu / \Lambda)^{-p / \nu_{2}-1} \gamma_{2}(\Lambda) / \Lambda$ where $p / \nu_{1}-1=p-1>0$ and $-p / \nu_{2}-$ $1=3 p-1 \rightarrow 2$ as $g_{12} \rightarrow 0$. It is confirmed that the point $(\infty, \infty)$ is another stable fixed point. In this case the scaling dimensions of the tunneling amplitudes are precisely equal to those of the instanton fugacities in the DIG picture. Hence we predict no crossover between the two pictures.

The whole $\left(u_{1}, u_{2}\right)$-plane is divided into two domains separated by a boundary (the dotted line in Fig.1), each of which has the attractive fixed point $(0, \infty)$ and $(\infty, \infty)$, respectively.

\section{DISCUSSION AND CONCLUSIONS}

In this paper we have shown that

(a) Scaling dimensions are universal only when both channels propagate in the same direction, which is guaranteed by requiring the stability of the system.

(b) In the presence of inter-channel tunnelings, the quasiparticle tunneling picture and the electron tunneling picture give different temperature dependence of the tunneling conductance and there exists a crossover between the two pictures.

(c) For the $(1,-1 / 3)$ state the phase diagram is divided into two domains giving different temperature dependence of the conductance.

Finally we explicitly write down our prediction for the temperature dependence of the tunneling conductance through a point contact in the presence of interactions.

First we consider the case where $\nu_{1} \nu_{2}>0(\mathrm{i})$. In the case of $\left(\nu_{1}, \nu_{2}\right)=(1 / 5,1 / 5)$ we can $\operatorname{read} \min \left[\alpha_{I J}\right]=-4 / 5, \delta_{D I G}=13$ and $\delta_{E T}=16 / 5$ from the tables of exponents. Hence

$$
G(T) \begin{cases}\propto T^{16 / 5} & (\text { at low temperature }) \\ \propto T^{13} & (\text { at intermediate temperature }) \\ =\frac{2}{5} \frac{e^{2}}{h}-\text { cst. } \times T^{-8 / 5} & \text { (at "high" temperature) }\end{cases}
$$

Similarly we obtain for the $(1 / 3,1 / 15)$ state

$$
G(T) \begin{cases}\propto T^{4} & \text { (at low temperature) } \\ \propto T^{5} & \text { (at intermediate temperature) } \\ =\frac{2}{5} \frac{e^{2}}{h}-\text { cst. } \times T^{-28 / 15} & \text { (at "high" temperature) }\end{cases}
$$

and for the $(1 / 3,1 / 3)$ state

$$
G(T) \begin{cases}\propto T^{4 / 3} & (\text { at low temperature) } \\ \propto T^{7} & \text { (at intermediate temperature) } \\ =\frac{2}{3} \frac{e^{2}}{h}-\text { cst. } \times T^{-4 / 3} & (\text { at "high" temperature) }\end{cases}
$$

Next we consider the $(1,-1 / 3)$ state which belongs to case (ii): $\nu_{1} \nu_{2}<0$. As was pointed out in the last section, our prediction is different for that state. There is an ambiguity with the quantized value of the Hall conductance at "high" temperature when two channels propagate 
in the opposite directions. In this case a generalized Landauer formula [16,28,29] gives different quantized values for the two-terminal conductance $G_{2}$ and the four-terminal Hall conductance $G_{H}$, which are both different from $\nu \frac{e^{2}}{h}$ [30,23]. For $\left(g_{+}, g_{-}\right)=\left(\nu_{1},\left|\nu_{2}\right|\right)=(1,1 / 3)$ they are calculated respectively as [30,23]

$$
G_{2}=\frac{e^{2}}{h}\left(g_{+}+g_{-}\right)=\frac{4}{3} \frac{e^{2}}{h}, \quad G_{H}=\frac{e^{2}}{h} \frac{g_{+}^{2}+g_{-}^{2}}{g_{+}-g_{-}}=\frac{5}{3} \frac{e^{2}}{h} .
$$

However we take the viewpoint that Landauer formula is no longer valid in this case and the conductance is quantized: $G(T) \rightarrow \frac{2}{3} \frac{e^{2}}{h}=\nu \frac{e^{2}}{h}$, which is experimentally observed.

As was studied in Secs. III and IV the RG flow diagram in the $\left(u_{1}, u_{2}\right)$-plane has two stable fixed points, which means that there are two different behaviors of the conductance depending on the initial value of $\left(u_{1}, u_{2}\right)$.

First we study the domain (a) of Fig. 1 where $\left(u_{1}, u_{2}\right) \rightarrow(0, \infty)$. If the initial value of $u_{1}$ is not so large only the quasihole $\left(\nu_{2}=-1 / 3\right)$ channel becomes reflected by the scattering potential as the temperature is lowered. The other $\left(\nu_{1}=1\right.$ integral quantum Hall) edge is robust against backward scatterings even at zero temperature. At zero temperature the conductance is again quantized. To obtain the quantized value, Landauer formula for the two-terminal [16,29] and four-terminal conductance was applied [17,28]. Similarly the two-terminal conductance can also be calculated [16,29]. Both $G_{2}$ and $G_{H}$ give the same value $\frac{e^{2}}{h}$ as $T \rightarrow 0$, which is identified with $G(T \rightarrow 0)$. At low temperature only $\theta_{1}^{(+)}$is relevant for the electron tunneling. In this case the Landauer formula is valid since the single-channel model is free from the complications on the equilibration between the channels [30,31].

The tunneling conductance behaves as

$$
G(T)= \begin{cases}\frac{e^{2}}{h}-c s t . \times T^{6 p-2} & \text { (at low temperature) } \\ \frac{e^{2}}{h}-c s t . \times T^{6 / p-2} & \text { (at intermediate temeprature) } \\ \frac{2}{3} \frac{e^{2}}{h}+c s t . \times T^{2 p / 3-2} & \text { (at "high" temperature) }\end{cases}
$$

where $p=\left(v_{1}-v_{2}\right) / g, g=\sqrt{\left(v_{1}-v_{2}\right)^{2}+4 \xi}$ and $\xi=\nu_{1} \nu_{2} g_{12}^{2}$. The signs of the temperature dependent terms reflect the fact that the $\nu_{2}=-1 / 3$ edge is that of a quasihole droplet. We predict the existence of crossover among the three pictures.

Next we study the domain (b) of Fig. 1 where $\left(u_{1}, u_{2}\right) \rightarrow(\infty, \infty)$. If the initial value of $u_{1}$ is sufficiently large both $u_{1}$ and $u_{2}$ scale to infinity. In this case the scaling dimension of the instanton fugacity $z_{1}$ in the DIG picture and that of the electron tunneling amplitude $\gamma_{1}$ are identical, i.e. bith give $p / \nu_{1}-1=p-1>0$. The tunneling conductance behaves at low temperature as

$$
G(T) \propto T^{2(p-1)}
$$

where $2(p-1)$ is a positive exponent if $g_{12}$ is finite but goes to zero as $g_{12} \rightarrow 0$. We expect that the exponent is small and there is no crossover between the ET and the DIG picture. In this case we also predict that even at "high" temperature the tunneling conductance is not quantized since this domain is not smoothly connected to the origin in the $\left(u_{1}, u_{2}\right)$-plane.

In summary we have shown that the structure of the hierarchical edge modes gives a variety of behaviors of the conductance through a point contact. This may give a clue to identify the edge structure in terms of the experiment. 


\section{ACKNOWLEDGMENTS}

We are grateful to A. Furusaki for useful discussions. This work was supported by a Grandin-Aid for Scientific Research No. 04240103 from the Ministry of Education, Science and Culture of Japan.

\section{APPENDIX A: BOSONIZATION OF THE EDGE MODE}

We start with the Lagrangian density

$$
\begin{aligned}
\mathcal{L} & =\frac{\nu}{4 \pi} \epsilon_{\mu \nu \lambda} a_{\mu} \partial_{\nu} a_{\lambda}+\Phi^{\dagger}\left\{i \partial_{0}-e\left(A_{0}+a_{0}\right)\right\} \Phi \\
& -\frac{1}{2 m}|\{-i \nabla+e(\vec{A}+\vec{a})\} \Phi|^{2}-V(|\Phi|)
\end{aligned}
$$

where $\Phi$ is a boson field and $a_{\mu}$ is a Chern-Simon gauge field. We assume that the interaction between the bosons is short-ranged: $V(|\Phi|)=\mu_{2}|\Phi|^{2}+\mu_{4}|\Phi|^{4}$ where $\mu_{2}$ is a chemical potential and $\mu_{4}$ comes from the Coulomb interaction. It is clear that the action is minimized by the trivial constant solution $\Phi=\sqrt{\bar{\rho}}$ with $\bar{\rho}=-\mu_{4} / 2 \mu_{2}$ and $A+a=\bar{A}+\bar{a}=0$. Using the equation of motion for $a_{\mu}$ and integrating over the density fluctuation $\delta \rho=\rho-\bar{\rho}$, we can write (A1) as

$$
\mathcal{L}=\frac{\nu}{4 \pi} \epsilon_{\mu \nu \lambda} \delta a_{\mu} \partial_{\nu} \delta a_{\lambda}+\frac{1}{4 \mu_{4}}\left\{\partial_{0} \theta+e\left(\delta A_{0}+\delta a_{0}\right)\right\}^{2}-\frac{\bar{\rho}}{2 m}\{\nabla \theta+e(\delta \vec{A}+\delta \vec{a})\}^{2}
$$

where $A=\bar{A}+\delta A$ and $a=\bar{a}+\delta a$. The single-valued part $\theta_{s}$ of the phase fluctuation $\theta$ is the Bogoliubov mode of the bose condensate whose sound velocity is given by $c^{2}=2 \mu_{4} \bar{\rho} / \mathrm{m}$, while the multi-valued part $\theta_{V}$ is the massive vortex excitation which, we assume, is absent in

the following discussions. Introducing the Stratonovich-Hubbard variable $J_{\mu}=\left(J_{0}, \vec{J}\right)$, we can write (A2) in the form

$$
\mathcal{L}=\frac{\nu}{4 \pi} \epsilon_{\mu \nu \lambda} \delta a_{\mu} \partial_{\nu} \delta a_{\lambda}-\frac{m}{2 \bar{\rho}}\left(c^{2} J_{0}^{2}-\vec{J}^{2}\right)-J_{\mu}\left\{\partial_{\mu} \theta_{s}+e\left(\delta A_{\mu}+\delta a_{\mu}\right)\right\}
$$

where $J_{\mu}$ is the fluctuation of the boson current from $(\bar{\rho}, \overrightarrow{0})$. Integration over $\theta_{s}$ gives the continuity equation for the boson current $\partial_{\mu} J_{\mu}=0$ which is automatically satisfied by writing $J_{\mu}$ in terms of the auxiliary gauge field $b_{\mu}$ as $J_{\mu}=\epsilon_{\mu \nu \lambda} \partial_{\nu} b_{\lambda} / 2 \pi$. After integrating out the gauge field fluctuation $\delta a$, we obtain

$$
\mathcal{L}=\frac{1}{4 \pi \nu} \epsilon_{\mu \nu \lambda} b_{\mu} \partial_{\nu} b_{\lambda} \operatorname{frac} 2 g\left(c^{2} f_{12}^{2}-f_{20}^{2}-f_{01}^{2}\right)-e J_{\mu} \delta A_{\mu}
$$

where $f_{\mu \nu}=\partial_{\mu} b_{\nu}-\partial_{\nu} b_{\mu}$ and $g=16 \pi^{2} \bar{\rho} / m$.

We consider a finite sample with both upper and lower edges. If two edges are well separated, we can assume that they are independent, and are described respectively by that of a semiinfinite plane $y<0(y>0)$ for the upper (lower) edge. We first turn off the additional electromagnetic field $\delta A$. The edge mode is a self-induced eigenmode of the FQH liquid which localizes near the boundary $y=0$ as $J_{\mu}(t, x, y)=\lambda e^{\lambda y} J_{\mu}^{(1 D)}(t, x)$ for $\mu=1,2$, where $J_{\mu}^{(1 D)}$ is the one-dimensional (1D) current, and $\lambda>0(\lambda<0)$ for the upper (lower) edge. In the absence of $\delta A$ we can assume $J_{2}(t, x, y)=0$ which is consistent with the boundary condition that the current does not flow outside the sample. Note that only with that condition the action remains gauge invariant [32]. Hence the current conservation reduces to $\partial_{0} J_{0}+\partial_{1} J_{1}=0$ which is satisfied 
by introducing the field $\phi^{(u, l)}$ for the upper (lower) edge such that $J_{0}^{(1 D)}(t, x)=\partial_{1} \phi^{(u, l)}(t, x) / 2 \pi$ and $J_{1}^{(1 D)}(t, x)=\mp \partial_{0} \phi^{(u, l)}(t, x) / 2 \pi$. We substitute these current densities into the equation of motion for $b_{\mu}$ derived from (A4). A solution is obtained when $\lambda= \pm g / 8 \pi \nu c$ with $J_{1}=\mp c J_{0}$, and $\left(\partial_{0} \pm c \partial_{1}\right) J_{0}=0$. The electron operator on the edges are written in terms of $\phi^{(u, l)}$ as

$$
\Psi^{(u, l)}(t, x, y=0)=\sqrt{\bar{\rho}} \exp \left( \pm i \phi^{(u, l)}(t, x) / \nu\right)
$$

which guarantees the relation between the $2 \mathrm{D}$ current and the phase of the electron operator: $\vec{J}^{(u, l)}(t, x, y=0)=(\bar{\rho} / m) \nabla \phi^{(u, l)}(t, x)$ [21]. Putting the 2D current into (A4), we obtain a Lagrangian density for a 1D boson:

$$
\mathcal{L}^{(1 D)(u, l)}=\int d y \mathcal{L}^{(2 D)}=\frac{c}{8 \pi \nu}\left\{\left(\frac{1}{c} \frac{\partial \phi^{(u, l)}}{\partial t}\right)^{2}+\left(\frac{\partial \phi^{(u, l)}}{\partial x}\right)^{2}\right\} \mp \phi^{(u, l)} \bar{E}_{1}
$$

where $\bar{A}_{\mu}(t, x)=\lambda \int d y e^{\lambda y} A_{\mu}(t, x, y)$. It should be noted that the existence of the Maxwell terms in the Lagrangian density (A4) is essential for our derivation. If two edges are well separated, we can write the total 1D Lagrangian density as a sum two chiral ones. It will be convenient to write the 1D Euclidean Lagrangian density as

$$
\mathcal{L}_{E}^{(\text {total })}=\frac{c}{8 \pi \nu}\left\{\left(\frac{\partial \phi^{(+)}}{\partial x}\right)^{2}+\left(\frac{\partial \phi^{(-)}}{\partial x}\right)^{2}\right\}+\frac{i}{4 \pi \nu} \frac{\partial \phi^{(+)}}{\partial \tau} \frac{\partial \phi^{(-)}}{\partial x}
$$

where $\phi^{( \pm)}=\phi^{(u)} \pm \phi^{(l)}$. Using the canonical quantization procedure, we can show $\left[J_{0}^{(u, l)}(k), J_{0}^{(u, l)}(-k)\right]= \pm \nu k / 2 \pi$ which is consistent with the anomaly equation $\partial_{0} J_{0}^{(1 D)}+$ $\partial_{1} J_{1}^{(1 D)}=(\nu / 2 \pi) E_{1}(t, x, y=0)$ [32].

\begin{tabular}{|c|c|c|}
\hline Exponents & (i) $\nu_{1} \nu_{2}>0$ & (ii) $\nu_{1} \nu_{2}<0$ \\
\hline$\alpha_{11}$ & $\left|\nu_{1}\right|-1$ & $\nu_{1} p-1$ \\
\hline$\alpha_{22}$ & $\left|\nu_{2}\right|-1$ & $-\nu_{2} p-1$ \\
\hline$\alpha_{12}$ & $\frac{\left|\nu_{1}\right|+\rho^{2}\left|\nu_{2}\right|}{2}-1$ & $\frac{\nu_{1}-\rho^{2} \nu_{2}}{2} p-\rho q-1$ \\
\hline Exponents & Universal & Non-universal \\
\hline
\end{tabular}

Table 1: $\alpha_{I J}$ 's are listed both for $\nu_{1} \nu_{2}>0$ (i) and for $\nu_{1} \nu_{2}<0$ (ii). They are universal only in case (i), otherwise depend on the interactions. Here $p$ and $q$ are given by $p=\left(v_{1}-v_{2}\right) / g, q=$ $2 \nu_{1} \nu_{2} g_{12} / g$ with $g=\sqrt{\left(v_{1}-v_{2}\right)^{2}+4 \xi}$ and $\rho=\nu_{1} / \nu_{2}$. Later we will define $\alpha_{12}$ as $\nu_{3} / 2-1$.

\begin{tabular}{|c|c|c|c|cc|}
\hline & \multicolumn{3}{|c|}{ (i) $\nu_{1} \nu_{2}>0$} & \multicolumn{2}{c|}{ (ii) $\nu_{1} \nu_{2}<0$} \\
\hline$\left(\nu_{1}, \nu_{2}\right)$ & $\left(\frac{1}{5}, \frac{1}{5}\right)$ & $\left(\frac{1}{3}, \frac{1}{15}\right)$ & $\left(\frac{1}{3}, \frac{1}{3}\right)$ & $\left(1,-\frac{1}{3}\right)$ & with $\left.g_{12} \rightarrow 0\right]$ \\
\hline$\nu$ & \multicolumn{2}{|c|}{$2 / 5$} & \multicolumn{3}{c|}{$2 / 3$} \\
\hline$\rho$ & 1 & 5 & 1 & -3 & {$[-3]$} \\
\hline$\alpha_{11}$ & $-4 / 5$ & $-2 / 3$ & $-2 / 3$ & $p-1>0$ & {$[0]$} \\
\hline$\alpha_{22}$ & $-4 / 5$ & $-14 / 15$ & $-2 / 3$ & $p / 3-1$ & {$[-2 / 3]$} \\
\hline$\alpha_{12}$ & $-4 / 5$ & 0 & $-2 / 3$ & $2 p-3 q-1>0$ & {$[1]$} \\
\hline
\end{tabular}

Table 2: Explicit values of $\alpha_{I J}$ 's are shown for the specific edge constructions with filling factors $\nu=2 / 5$ and $\nu=2 / 3$. Note that most of the potentials are relevant. Some exceptions are interchannel tunnelings in the case of $\left(\nu_{1}, \nu_{2}\right)=(1 / 3,1 / 15)$ and $(1,-1 / 3)$. In both cases $\rho$ takes a large absolute value, which blocks inter-channel tunnelings. The other exception is the $U_{11}$ in the case of $\left(\nu_{1}, \nu_{2}\right)=(1,-1 / 3)$. The process is marginal in the absence of inter-channel interactions, but becomes irrelevant in the presence of infinitesimal $g_{12}$ since $p>1$. 


\begin{tabular}{|c|c|c|c|c|c|c|c|c|c|}
\hline$I \backslash j$ & 1 & 2 & 3 & 4 & 5 & 6 & 7 & 8 & 9 \\
\hline 1 & 1 & 1 & 0 & 0 & 1 & -1 & 2 & 0 & 0 \\
\hline 2 & 1 & -1 & 1 & 1 & 0 & 0 & 0 & 2 & 0 \\
\hline 3 & 0 & 0 & 1 & -1 & 1 & 1 & 0 & 0 & 2 \\
\hline
\end{tabular}

Table 3: $C_{I j}$ 's are listed. I specifies the "coordinate" in the $\left(\theta_{1}, \theta_{2}, \theta_{3}\right)$-space, and $j$ denotes the species of an instanton or an anti-instanton.

\begin{tabular}{|c|c|c|}
\hline & $\left(\right.$ i) $\nu_{1} \nu_{2}>0$ & (ii) $\nu_{1} \nu_{2}<0$ \\
\hline$\nu_{3}$ & $\left|\nu_{1}\right|+\rho^{2}\left|\nu_{2}\right|$ & $\left(\nu_{1}-\rho^{2} \nu_{2}\right) p-2 \rho q$ \\
\hline$\beta_{1,2}$ & $\frac{1}{\left|\nu_{1}\right|}+\frac{1}{\left|\nu_{2}\right|}-1$ & $\left(\frac{1}{\nu_{1}}-\frac{1}{\nu_{2}}\right) p \pm \frac{2 q}{\nu_{1} \nu_{2}}-1$ \\
\hline$\beta_{3}=\beta_{4}$ & $\frac{1}{\left|\nu_{2}\right|}+\frac{1}{\nu_{3}}-1$ & $-\frac{p}{\nu_{2}}+\frac{1}{\nu_{3}}-1$ \\
\hline$\beta_{5}=\beta_{6}$ & $\frac{1}{\nu_{3}}+\frac{1}{\left|\nu_{1}\right|}-1$ & $\frac{1}{\nu_{3}}+\frac{p}{\nu_{1}}-1$ \\
\hline$\beta_{7}$ & $\frac{4}{\left|\nu_{1}\right|}-1$ & $\frac{4 p}{\nu_{1}}-1$ \\
\hline$\beta_{8}$ & $\frac{4}{\left|\nu_{2}\right|}-1$ & $-\frac{4 p}{\nu_{2}}-1$ \\
\hline$\beta_{9}$ & $\frac{4}{\nu_{3}}-1$ & $\frac{4}{\nu_{3}}-1$ \\
\hline Exponents & Universal & Non-universal \\
\hline
\end{tabular}

Table 4: $\beta_{j}$ 's are listed both for $\nu_{1} \nu_{2}>0$ (i) and for $\nu_{1} \nu_{2}<0$ (ii). They are universal only in case (i), otherwise depend on the interactions.

\begin{tabular}{|c|c|c|c|cc|}
\hline & \multicolumn{2}{|c|}{$(1) \nu_{1} \nu_{2}>0$} & \multicolumn{2}{c|}{$(2) \nu_{1} \nu_{2}<0$} \\
\hline$\left(\nu_{1}, \nu_{2}\right)$ & $\left(\frac{1}{5}, \frac{1}{5}\right)$ & $\left(\frac{1}{3}, \frac{1}{15}\right)$ & $\left(\frac{1}{3}, \frac{1}{3}\right)$ & $\left(1,-\frac{1}{3}\right)$ & [with $\left.g_{12} \rightarrow 0\right]$ \\
\hline$\nu$ & \multicolumn{2}{|c|}{$2 / 5$} & \multicolumn{3}{|c|}{$2 / 3$} \\
\hline$\rho$ & 1 & 5 & 1 & -3 & {$[-3]$} \\
\hline$\nu_{3}$ & $2 / 5$ & 2 & $2 / 3$ & $4 p-6 q$ & {$[4]$} \\
\hline$\beta_{1,2}$ & 9 & 17 & 5 & $4 p \mp 6 q-1$ & {$[3]$} \\
\hline$\beta_{3}=\beta_{4}$ & $\frac{13}{2}^{\dagger}$ & $\frac{29}{2}$ & $\frac{7}{2}^{\dagger}$ & $3 p+\frac{1}{4 p-6 q}-1$ & {$\left[\frac{9}{4}\right]$} \\
\hline$\beta_{5}=\beta_{6}$ & $\frac{13}{2}^{\dagger}$ & $\frac{5}{2}^{\dagger}$ & $\frac{7}{2}^{\dagger}$ & $\frac{1}{4 p-6 q}+p-1$ & {$\left[\frac{1}{4}\right]$} \\
\hline$\beta_{7}$ & 19 & 11 & 11 & $4 p-1$ & {$[3]$} \\
\hline$\beta_{8}$ & 19 & 59 & 11 & $12 p-1$ & {$[11]$} \\
\hline$\beta_{9}$ & 9 & 1 & 5 & $\frac{4}{4 p-6 q}-1<0$ & {$[0]$} \\
\hline
\end{tabular}

Table 5: $\beta_{j}$ 's are evaluated for the specific edge constructions. Minima of $\beta_{j}$ 's are marked by a superscript $\dagger$. For the $(1,-1 / 3)$ state $\beta_{9}<0$, i.e. $y_{9}$ scales to infinity when $g_{12}$ is finite. The instanton picture gives the correct temperature dependence of the tunneling conductance only when all $y_{j}$ 's are irrelevant, i.e. all the original scattering potentials scale to infinity.

\begin{tabular}{|l|c|c|c|}
\hline Inter-channel & \multicolumn{3}{|c|}{$\left(\nu_{1}, \nu_{2}\right)$} \\
\cline { 2 - 4 } tunnelings & $\left(\frac{1}{5}, \frac{1}{5}\right)$ & $\left(\frac{1}{3}, \frac{1}{15}\right)$ & $\left(\frac{1}{3}, \frac{1}{3}\right)$ \\
\hline absent & 8 & 4 & 4 \\
\hline present & 13 & 5 & 7 \\
\hline
\end{tabular}

Table 6: Explicit values of $\delta_{E T}$ 's are shown both in the presence and in the absence of interchannel tunnelings. 
Fig.1

The whole $\left(u_{1}, u_{2}\right)$-plane is divided into two domains separated by a boundary (the dotted line), each of which has the attracive fixed point $(0, \infty)(a)$ and $(\infty, \infty)(b)$, respectively. The former (a) corresponds to the picture where only quasihole channel is reflected, and the latter (b) to the one where both channels are reflected. 


\section{REFERENCES}

[1] X.G. Wen, Phys. Rev. B41, 12838 (1990).

[2] C.L. Kane and M.P.A. Fisher, Phys. Rev. B46, 15233 (1992).

[3] A. Furusaki and N. Nagaosa, Phys. Rev. B47, 3827 (1993).

[4] X.G. Wen, Phys. Rev. B44, 5708 (1991).

[5] X.G. Wen, Int. J. Mod. Phys. B6, 1711 (1992).

[6] P. Fendley, A.W.W. Ludwig and H. Saleur, Phys. Rev. B52, 8934 (1995).

[7] F.P. Milliken, C.P. Umbach, and R.A. Webb, Solid State Commun. 97, 309 (1996).

[8] K. Moon, H. Yi, C.L. Kane, S.M. Girvin, and M.P.A. Fisher, Phys. Rev. Lett. 71, 4381 (1993).

[9] F. D. M. Haldane, Phys. Rev. Lett. 51, 605 (1983).

[10] B. I. Halperin, Phys. Rev. Lett. 52, 1583 (1984).

[11] B. Blok and X.G. Wen, Phys. Rev. B42, 8133 (1990).

[12] J. K. Jain, Phys. Rev. Lett. 63, 199 (1989)

[13] J. K. Jain, Phys. Rev. B40, 8079 (1989)

[14] B. Blok and X.G. Wen, Phys. Rev. B42, 8145 (1990).

[15] D.B. Chklovski, Phys. Rev. B51, 9895 (1995).

[16] C.W.L. Beenakker, Phys. Rev. Lett. 64, 216 (1990).

[17] A.H. MacDonald, Phys. Rev. Lett. 64, 220 (1990).

[18] L. Brey, Phys. Rev. B50, 11861 (1994).

[19] P.A. Maksym, J. Phys. C1, 6299 (1989)

[20] D.A. Syphers and J.E. Furneaux, Surf. Sci. 196, 252 (1988).

[21] N. Nagaosa and M. Kohmoto, in Correlation Effects in Low-Dimensional Electron Systems, edited by A. Okiji and N. Kawakami (Springer-verlag, Berlin, 1994) p. 168.

[22] X.G. Wen, Phys. Rev. B43, 11025 (1991).

[23] C.L. Kane and M.P.A. Fisher, preprint cond-mat/9409028).

[24] X.G. Wen, preprint (cond-mat/9506066).

[25] M.P.A. Fisher and W. Zwerger, Phys. Rev. B32, 6190 (1985).

[26] A. Schmid, Phys. Rev. Lett. 51, 1506 (1983).

[27] G.D. Mahan, Many-Particle Physics, Chap.9 (Plenum, New York,1981).

[28] M. Buttiker, Phys. Rev. Lett. 57, 1761 (1986).

[29] M. Buttiker, Phys. Rev. B38, 9375 (1988).

[30] C.L. Kane, M.P.A. Fisher and J. Polchinski Phys. Rev. Lett. 72, 4129 (1994).

[31] Y. Oreg and M. Finkel'stein, Phys. Rev. Lett. 74, 3668 (1995).

[32] N. Nagaosa and M. Kohmoto, Phys. Rev. Lett. 75, 4294 (1995). 


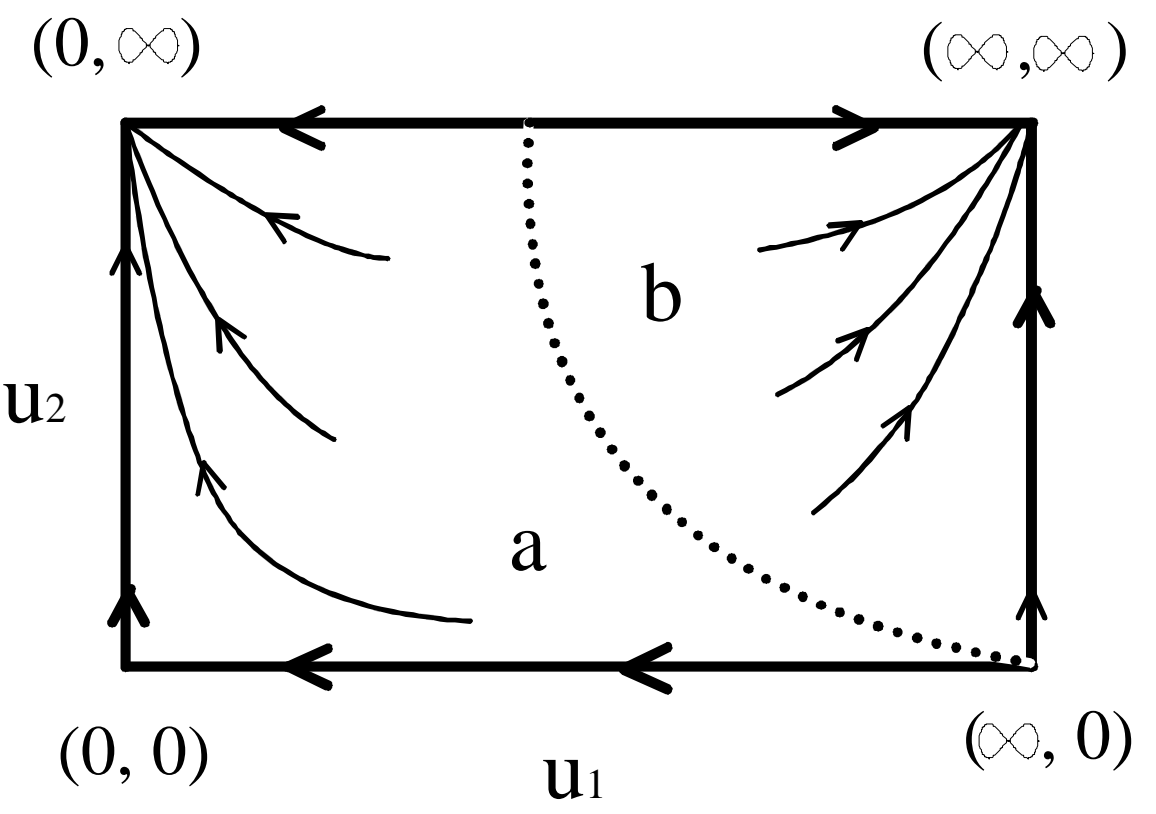

